OPEN ACCESS

Edited by:

Yoav Keynan

University of Manitoba, Canada

Reviewed by:

Retno Wahyuningsih,

Universitas Kristen

Indonesia, Indonesia

Yanhui Chen,

GeneCast Biotechnology Co.

Ltd., China

*Correspondence:

Kai Deng

dengkaik@gmail.com

Specialty section

This article was submitted to Infectious Diseases - Surveillance,

Prevention and Treatment,

a section of the journal

Frontiers in Medicine

Received: 26 June 2021 Accepted: 01 November 2021 Published: 22 November 2021

Citation:

Wang Y and Deng K (2021)

Environmental Risk Factors for Talaromycosis Hospitalizations of HIV-Infected Patients in Guangzhou,

China: Case Crossover Study.

Front. Med. 8:731188.

doi: 10.3389/fmed.2021.731188

\section{Environmental Risk Factors for Talaromycosis Hospitalizations of HIV-Infected Patients in Guangzhou, China: Case Crossover Study}

\author{
Yaping Wang and Kai Deng* \\ Infectious Diseases Institute, Guangzhou Eighth People's Hospital, Guangzhou Medical University, Guangzhou, China
}

Talaromycosis is a fatal opportunistic infection prevalent in human immunodeficiency virus (HIV)-infected patients, previous studies suggest environmental humidity is associated with monthly talaromycosis hospitalizations of HIV-infected patients, but the acute risk factor remains uncertain. In this study, we evaluated the associations between talaromycosis hospitalizations of HIV-infected patients $(n=919)$ and environmental factors including meteorological variables and air pollutants at the event day (assumed "lag 0" since the exact infection date is hard to ascertain) and 1-7 days prior to event day (lag 1-lag 7) in conditional logistics regression models based on a case crossover design. We found that an interquartile range (IQR) increase in temperature at lag 0-lag 7 (odds ratio [OR] [95\% Cl] ranged from 1.748 [1.345-2.273] to 2.184 [1.672-2.854]), and an IQR increase in humidity at lag 0 (OR [95\% Cl] = 1.192 [1.052-1.350]), and lag 1 $(\mathrm{OR}[95 \% \mathrm{Cl}]=1.199$ [1.056-1.361]) were significantly associated with talaromycosis hospitalizations of HIV-infected patients. Besides, temperature was also a common predictor for talaromycosis in patients with co-infections including candidiasis $(n=386)$, Pneumocystis pneumonia $(n=183)$, pulmonary tuberculosis $(n=141)$, and chronic hepatitis $(n=158)$, while humidity was a specific risk factor for talaromycosis in patients with candidiasis, and an air pollutant, $\mathrm{SO}_{2}$, was a specific risk factor for talaromycosis in patients with Pneumocystis pneumonia. In an age stratified evaluation (cutoff $=50$ years old), temperature was the only variable positively associated with talaromycosis in both younger and older patients. These findings broaden our understanding of the epidemiology and pathogenesis of talaromycosis in HIV-infected patients.

Keywords: talaromycosis, temperature, humidity, $\mathrm{SO}_{2}$, $\mathrm{HIV}$

\section{INTRODUCTION}

Talaromycosis is one of the most prevalent opportunistic infections among human immunodeficiency virus (HIV)-infected patients in South and Southeast Asia (1-3), and talaromycosis cases outside endemic areas were also reported partially due to increasing migration and travel to endemic regions, and the causes for some cases in non-endemic regions remain enigmatic (4-6). The prevalence of talaromycosis is closely associated with HIV infection $(2,7)$, e.g., in mainland China, $87.72 \%$ of talaromycosis occurs in HIV-infected patients (3). HIV-infected patients with a CD4 count lower than 100 cells per $\mu \mathrm{L}$ are susceptible to Talaromyces marneffei, 
the pathogen of talaromycosis, and have a high risk of death $(8-10)$. The common clinical manifestations of talaromycosis include fever, weight loss, anemia, lymphadenopathy, and skin lesions $(3,10)$, and concomitant infections are frequent, particularly tuberculosis and salmonella infection $(11,12)$. The in-hospital mortality of HIV patients with talaromycosis was $17.5 \%$ in southern China, significantly higher than those without talaromycosis (7.6\%) (9).

Although Talaromyces marneffei was first isolated from a bamboo rat early in 1956 and its mycology was described almost at the same time (2), knowledge regarding critical issues in talaromycosis epidemiology is still lacking. To date, there is no evidence of animal-to-human or human-to-human spread of talaromycosis. Thus, how a human host becomes infected remains elusive, and it was proposed that exposure to an unidentified common environmental reservoir may represent the transmission route $(13,14)$. Occupational exposure to soil, rainy season were considered as risk factors for talaromycosis, while consumption of bamboo rat was not (15). It was reported that a meteorological factor, humidity, was the most important predictor for monthly talaromycosis hospitalizations of HIV-infected patients in Vietnam based on Poisson regression analyses (16). However, whether meteorological factors have an acute effect on talaromycosis events remains uncertain. Besides, air pollutants have attracted more and more attention due to their significant associations with various infectious diseases including Pneumocystis pneumonia, an opportunistic infection in HIV patients $(17,18)$. Unfortunately, to our knowledge, by now there is no study regarding the associations between air pollutants and talaromycosis in HIV-infected patients.

Guangzhou is located in southern China with a humid subtropical climate. Guangzhou Eighth People's Hospital is the largest infectious disease hospital in Guangzhou and the training base for clinicians in HIV and acquired immune deficiency syndrome (AIDS). More than $80 \%$ of HIV-infected patients in Guangzhou were treated here during the last decade according to Guangzhou Centers for Disease Control and Prevention (CDC) reports (19). The main goal of the present study was to determine the acute associations between environmental factors including meteorological variables and air pollutants, and talaromycosis hospitalizations of HIV-infected patients based on a case crossover design.

\section{MATERIALS AND METHODS}

\section{Study Population}

This study followed the guideline of the Ethics Committee of Guangzhou Eighth People's Hospital. The individual identifiers were not used in our study, so informed consent was not specifically required. We extracted information on the hospitalization date, diagnosis, CD4 cell count, age, sex from the records from January 1, 2014, through December 31, 2019. The patients who resided outside Guangzhou were excluded. Talaromycosis diagnosis was identified based on the International Classification of Diseases, 10th revision (ICD-10) codes B48.4, and confirmed by isolation of Talaromyces marneffei from bone marrow with standard culture techniques (16). In case of repeated hospitalizations, only the first event was included for analyses. Since the pathogens of co-infections among HIVinfected patients are usually part of the human saprophytic microflora, the diagnosis of co-infection was based on medical history, symptoms, physical examinations, and laboratory tests. To confirm Cryptococcus neoformans co-infection, body fluid (blood, cerebrospinal fluid, or sputum) was collected for culture, and a chest X-ray or computerized tomography scan of lungs, brain, or other parts of the body was conducted if necessary; for the diagnosis of candidiasis, samples from the infected body site (oral, skin, or vaginal discharge), blood, or lung biopsy were used for culture; sputum or bronchoalveolar lavage was collected for diagnosing Pneumocystis pneumonia; the diagnosis of pulmonary tuberculosis was established by isolation of mycobacterium tuberculosis from sputum or bronchoalveolar lavage.

\section{Meteorological and Air Pollution Data}

Daily meteorological data, including temperature, humidity, wind speed, and pressure, were collected from Weather Underground, IBM (https://www.wunderground.com) from January 1, 2014, through December 31, 2019. Historical daily air pollution data, including coarse particulate matter $\left(\mathrm{PM}_{10}\right)$, sulfur dioxide $\left(\mathrm{SO}_{2}\right)$, carbon monoxide $(\mathrm{CO})$, nitrogen dioxide $\left(\mathrm{NO}_{2}\right)$, and ozone $\left(\mathrm{O}_{3}\right)$ were obtained from the National Air Quality Study Platform (https://www.aqi study.cn).

\section{Statistics Analysis}

To analyze talaromycosis events in HIV patients, we employed a case crossover design. In this approach, the day of hospitalization date (event day, lag 0), and 1-7 days prior to event day (lag $1-\operatorname{lag} 7)$ were examined separately $(20,21)$. For each lag day,

TABLE 1 | Demographic and clinical features of 919 HIV-infected patients with talaromycosis.

\begin{tabular}{lc}
\hline Demographic characteristic & Value \\
\hline Male & $751(81.7 \%)$ \\
Age, years & $39.6(30-48)$ \\
CD4 cell count, cells/ $\mu$ L & $9(4-21)$ \\
Co-infection & \\
C. neoformans infection & $29(3.2 \%)$ \\
Candidiasis & $386(42.0 \%)$ \\
Pneumocystis pneumonia & $183(19.9 \%)$ \\
Pulmonary tuberculosis & $141(15.3 \%)$ \\
Chronic hepatitis B or C & $158(17.2 \%)$ \\
Comorbidity & \\
Diabetes & $21(2.3 \%)$ \\
Liver cirrhosis & $22(2.4 \%)$ \\
\hline
\end{tabular}

Data are in absolute count (\%) for categorical variables and median (interquartile range [IQR]) for continuous data. 
the control days were usually defined as the days on the same day of the week in the same month and year. This control strategy aiming to adjust for the effects of time-dependent confounding variables has been widely applied to evaluate acute risk factors for health outcomes $(18,22-24)$. Given that the incubation period of talaromycosis is $0-3$ weeks (16), we adopted 21 and 28 days prior to event day as control days in this study.

To explore the environmental variables potentially associated with talaromycosis hospitalizations, we conducted a conditional logistics regression analysis. Odds ratios (OR) and $95 \%$ confidence interval (CI) were estimated for an interquartile range (IQR) increase in each environmental variable. The variables with $P<0.05$ in univariate analysis were combined for multivariate analysis using the forward stepwise (likelihood ratio) method, and the variables with $P<0.05$ were considered significant. All the statistical analyses were performed using IBM SPSS Statistics 26 (USA). Graphic representations were performed with GraphPad Prism 8 software.

\section{RESULTS}

\section{Study Subjects and Environmental Data}

From January 2014 to December 2019, 919 consecutive HIV patients were admitted to Guangzhou Eighth People's Hospital with Talaromycosis (Table 1). All the patients had received antiviral therapy before admission. The median age of hospitalized patients was 39.6 years, and $81.7 \%(n=751)$ were male. The median CD4 cell count was 9 cells/ $\mu \mathrm{L}$ on admission. The most frequent co-infection was candidiasis (42.0\%, including 381 oral candidiasis, 3 Candida pneumonia, 1 cutaneous candidiasis, and 1 vaginal candidiasis), followed by Pneumocystis pneumonia (19.9\%), chronic hepatitis B or C (17.2\%), pulmonary tuberculosis (15.3\%), and Cryptococcus neoformans infection (3.2\%). The main comorbidities included liver cirrhosis (2.4\%) and diabetes (2.3\%).

The summary statistics for environmental variables from 2014 to 2019 were reported in Supplementary Table S1. There was a positive correlation between air pollutants $\left(\mathrm{PM}_{10}, \mathrm{SO}_{2}\right.$, $\mathrm{CO}, \mathrm{NO}_{2}$, and $\mathrm{O}_{3}$ ) except that $\mathrm{CO}$ was negatively correlated
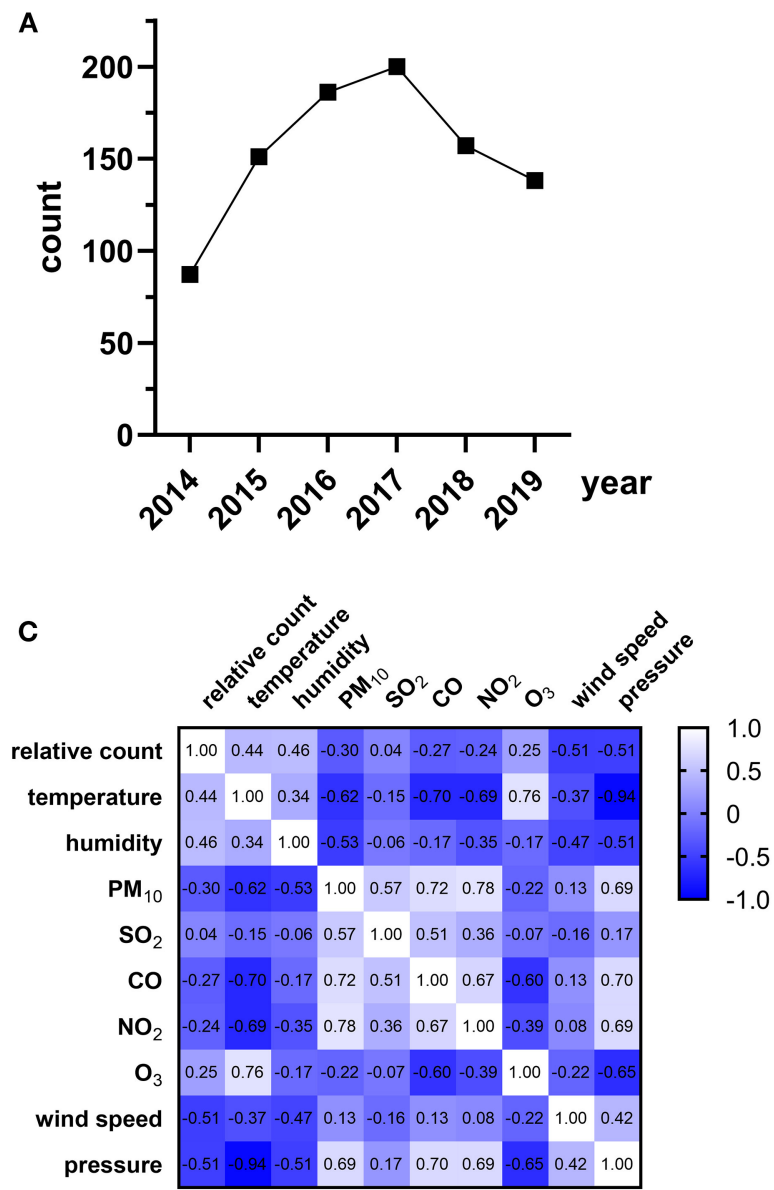

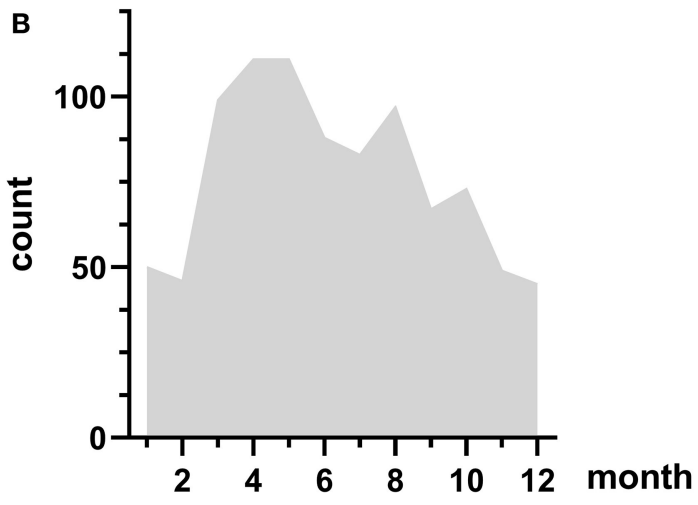

D

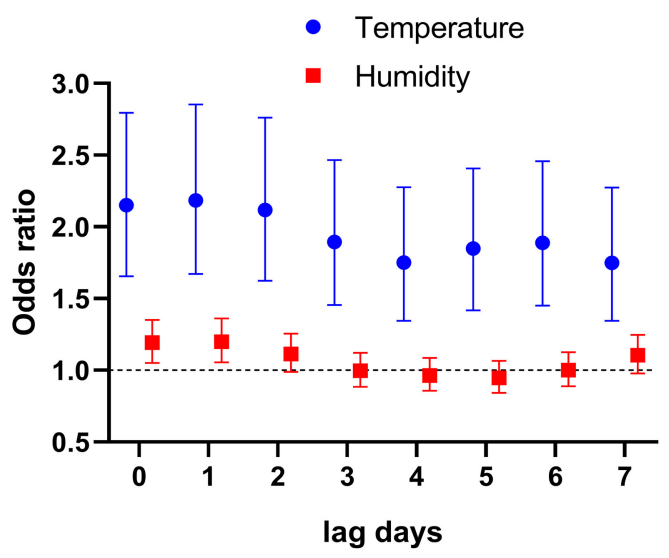

FIGURE 1 | (A) Yearly talaromycosis hospitalizations of HIV-infected patients during 2014-2019. (B) Average monthly talaromycosis hospitalizations of HIV-infected patients. (C) Spearman's correlations between relative count of monthly talaromycosis hospitalizations and environmental variables. The correlation coefficient is shown in each cell. (D) Environmental variables significantly associated with talaromycosis hospitalizations. 
to $\mathrm{O}_{3}(r=-0.17$, Supplementary Figure $\mathrm{S} 1)$. Temperature was positively associated with $\mathrm{O}_{3}(r=0.50)$ and negatively associated with other air pollutants. Humidity was positively and weakly correlated to CO and temperature $(r=0.09$ and 0.16 , respectively) and negatively correlated to other variables.

\section{Effects of Short Term Exposure to Environmental Variables on Talaromycosis Hospitalizations}

The HIV admissions in Guangzhou Eighth People's Hospital increased rapidly from 1,395 to 2,767 during the study period, while the trend of talaromycosis hospitalizations of HIV-infected patients was not always consistent, which increased from 87 in 2014 to a peak $(n=200)$ in 2017 , and gradually decreased to 138 in 2019 (Figure 1A). There were more talaromycosis hospitalizations during the warm season (average monthly hospitalizations $=91$, from March to October) than the cold season (average monthly hospitalizations $=48$, from November to February, Figure 1B). We defined the percentage of monthly hospitalizations counts in the same year as relative count, and found it was moderately and positively correlated to temperature $(r=0.44)$ and humidity $(r=0.46$, Figure 1C and Supplementary Figure S2), and weakly correlated to $\mathrm{O}_{3}(r$ $=0.25$ ).

To explore the environmental variables that associated with talaromycosis hospitalizations, we conducted a univariate analysis first and only the variables significant in univariate model $(P<0.05)$ were combined for multivariate analyses. The results were reported in Supplementary Table S2, and the environmental variables significantly associated with talaromycosis hospitalizations were shown in Figure 1D, that an IQR increase in temperature at lag $0(\mathrm{OR}[95 \% \mathrm{CI}]=2.151$ [1.656-2.794]), lag 1 (OR [95\% CI] = 2.184 [1.672-2.854]), lag $2(\mathrm{OR}[95 \% \mathrm{CI}]=2.117[1.623-2.761])$, lag $3(\mathrm{OR}[95 \% \mathrm{CI}]$ $=1.895[1.456-2.466])$, lag $4(\mathrm{OR}[95 \% \mathrm{CI}]=1.750[1.345-$ $2.277])$, lag 5 (OR [95\% CI] $=1.848[1.418-2.408])$, lag $6(\mathrm{OR}$ $[95 \% \mathrm{CI}]=1.888[1.450-2.458])$, and lag 7 (OR $[95 \% \mathrm{CI}]=$ $1.748[1.345-2.273])$, and an IQR increase in humidity at lag $0(\mathrm{OR}[95 \% \mathrm{CI}]=1.192[1.052-1.350])$ and lag1 (OR $[95 \%$ $\mathrm{CI}]=1.199$ [1.056-1.361]) were significantly associated with talaromycosis hospitalizations of HIV-infected patients, while an IQR increase in humidity from lag 2 to lag 7 were not associated with talaromycosis event. These data suggested that temperature and humidity represent the acute risk factors for talaromycosis in HIV-infected patients.

\section{Risk Factors for Talaromycosis Hospitalizations of HIV-Infected Patients With Co-infections}

One of the most striking clinical features of HIV-infected patients is various co-infections including opportunistic infections, which are the leading cause of AIDS-related death $(25,26)$. In our
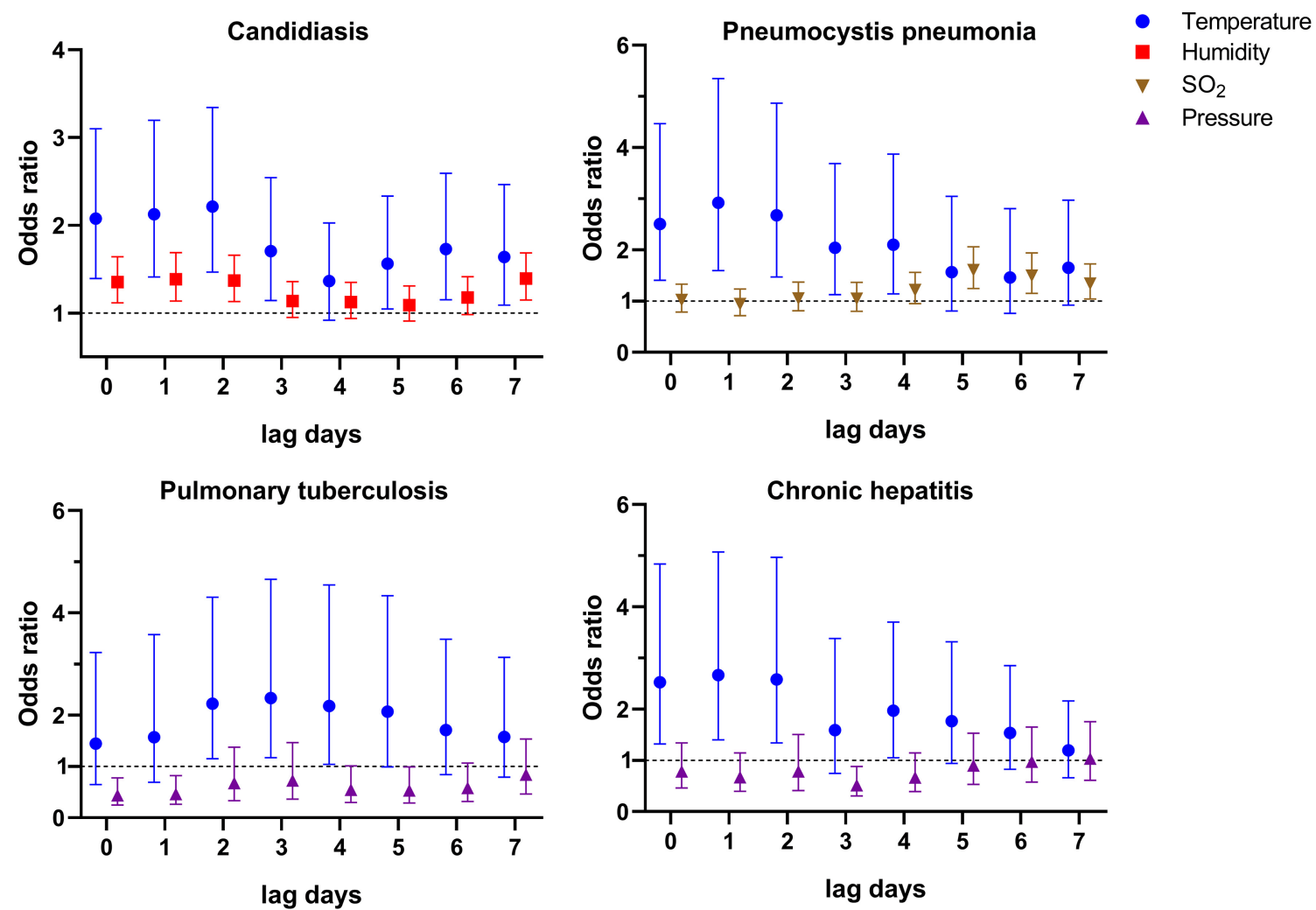

FIGURE 2 | Environmental variables significantly associated with talaromycosis hospitalizations of HIV-infected patients with co-infections. 
cohort, there were approximately half of patients with candidiasis (42.0\%), and nearly $1 / 5$ of patients with Pneumocystis pneumonia (19.9\%), chronic hepatitis B or C $(17.2 \%)$, and pulmonary tuberculosis (15.3\%). Thus, we also evaluated the risk factors for talaromycosis in patients with the four main co-infections (Supplementary Tables S3-S6), and the variables significantly associated with talaromycosis were shown in Figure 2. For patients with candidiasis, an IQR increase in temperature at lag 0-lag 3, and lag 5-lag 7 (OR [95\% CI] ranged from 1.564 [1.048-2.332] to 2.214 [1.467-3.341]), and an IQR increase in humidity at lag 0-lag 2, and lag 7 (OR [95\% CI] ranged from 1.354 [1.117-1.642] to $1.393[1.150-1.687])$ were significantly associated with talaromycosis; for patients with Pneumocystis pneumonia, an IQR increase in temperature at lag 0-lag 4 (OR [95\% CI] ranged from $2.040[1.128-3.689]$ to 2.924 [1.598$5.348]$ ), and an IQR increase in an air pollutant, $\mathrm{SO}_{2}$, at lag 5 (OR $[95 \% \mathrm{CI}]=1.605[1.249-2.064])$, lag $6(\mathrm{OR}[95 \% \mathrm{CI}]=1.495$ [1.151-1.943]), and lag 7 (OR [95\% CI] $=1.342$ [1.043-1.726]) were significantly associated with talaromycosis; for patients with pulmonary tuberculosis, an IQR increase in temperature at lag 2-lag 4 (OR [95\% CI] ranged from 2.180 [1.045-4.546] to 2.339 [1.174-4.659]) were positively associated with talaromycosis, while an IQR increase in pressure at lag 0 -lag 1 , and lag 5 (OR [95\% CI] ranged from 0.439 [0.248-0.779] to 0.534 [0.287-0.991]) were negatively associated with talaromycosis; for patients with chronic hepatitis, an IQR increase in temperature at lag $0-\operatorname{lag} 2$, and lag $4(\mathrm{OR}[95 \% \mathrm{CI}]$ ranged from 1.973 [1.051$3.704]$ to 2.666 [1.401-5.074]) were positively associated with talaromycosis, while an IQR increase in pressure at lag 3 (OR $[95 \% \mathrm{CI}]=0.519[0.305-0.882])$ was negatively associated with talaromycosis. These results revealed temperature as a common predictor, as well as distinct risk factors for talaromycosis in HIV-infected patients with various co-infections.

\section{Risk Factors for Talaromycosis Hospitalizations of HIV-Infected Patients Stratified by Age}

There are more and more elderly HIV-infected patients due to the improvements of antiretroviral therapy, e.g., the HIV-infected patients older than 50 years in the United States had increased to $51 \%$ in 2018 (27). However, the specific risk factors for talaromycosis in younger and older patients remain uncertain, thus, we here conducted an age stratified evaluation. In our cohort, there were 190 patients aged 50 and above (defined as "older patients"), and 729 patients younger than 50 years (defined as "younger patients"). The demographic and clinical data showed that there was no significant difference in gender distribution, CD4 cell count, and the frequency of co-infections and liver cirrhosis between younger and older patients, and more diabetes (younger vs. older: 1.5 vs. $5.3 \%, P=0.005$ ) in the older patients (Supplementary Table S7). Multivariate logistics regression results showed an IQR increase in temperature at lag 0 -lag 7 (OR [95\% CI] ranged from 1.598 [1.190-2.146] to 2.214 [1.636-2.994]) were significantly associated with talaromycosis in younger patients (Supplementary Table S8 and Figure 3); for older patients, an IQR increase in temperature at lag 5-lag 7
(OR [95\% CI] ranged from $2.441[1.364-4.368]$ to 3.419 [1.8856.201]) were positively associated with talaromycosis, while an IQR increase in pressure at lag 0-lag $4(\mathrm{OR}[95 \% \mathrm{CI}]$ ranged from 0.439 [0.259-0.745] to 0.515 [0.304-0.872]) were negatively associated with talaromycosis (Supplementary Table S9 and Figure 3). These data suggested that temperature was the common risk factor for talaromycosis in both younger and older patients, and pressure was a negative predictor for talaromycosis in older patients.

\section{DISCUSSION}

Previous studies have suggested meteorological variables like rainy season (12) and humidity (16) as predictors for monthly talaromycosis hospitalizations of HIV-infected patients. However, to our knowledge, the acute risk factors for talaromycosis are still elusive and warrant further investigation. Here, based on a case crossover design, we showed an IQR increase in humidity at lag 0 and lag 1 were significantly associated with talaromycosis hospitalizaitons of HIV-infected patients in Guangzhou, China (Figure 1D and Supplementary Table S2), consistent with previous findings. It was proposed that humidity may facilitate inhalation of infectious spores or hyphal fragment from the environmental reservoir driving talaromycosis pathogenesis $(2,16)$, our study adds new evidence of the theory. Besides, we also found an IQR increase in another meteorological variable, temperature, at lag 0 -lag 7 were significantly associated with talaromycosis (Figure 1D). We noted that in the previous study conducted in Ho Chi Minh, Vietnam, temperature was not associated with monthly talaromycosis hospitalizations (16). This discrepancy may be explained by the distinct acute and subacute risk factors for talaromycosis, and different geographic regions. Interestingly, temperature was also considered as one of the most important variables in a maxent model recently established by Wei et al. (28) for predicting the distribution of global talaromycosis, supporting the results of our study. The median of $\mathrm{CD} 4$ counts in our cohort, which are closely related to talaromycosis pathogenesis $(8-10)$, was as low as 9 cells $/ \mu \mathrm{L}$. Therefore, our cohort represented a population susceptible to talaromycosis.

We also showed that temperature was a common acute risk factor for talaromycosis in HIV-infected patients with four main co-infections, candidiasis, Pneumocystis pneumonia, pulmonary tuberculosis, and chronic hepatitis (Figure 2). By contrast, humidity was only associated with talaromycosis in patients with candidiasis but not other co-infections. It was reported that a humid environment favors the growth of Candida species, the pathogen of candidiasis, and promotes candidiasis pathogenesis (29). Thus, it seems that humidity is a common risk factor for talaromycosis and candidiasis. Another possible explanation is that a humid environment promotes candidiasis, and the latter is associated with talaromycosis progress, as we found humidity was not associated with talaromycosis in patients with the other three co-infections (Figure 2). Approximately half of the patients (42.0\%) in our cohort were 

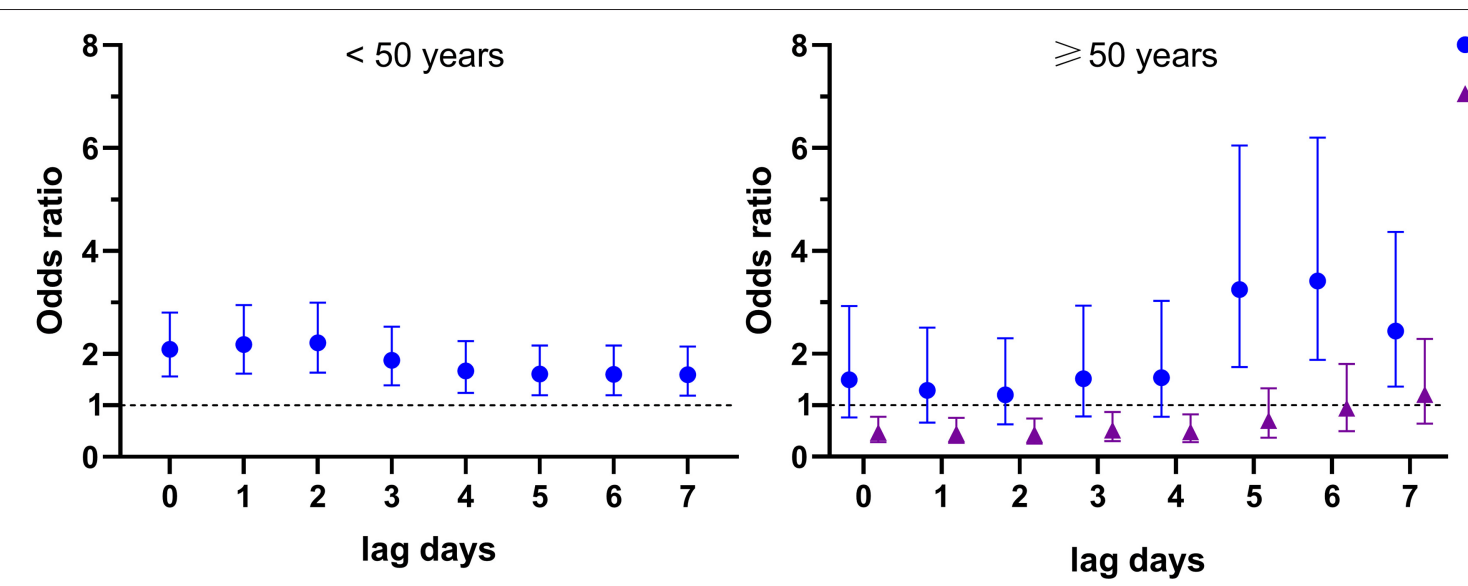

- Temperature

- Pressure

FIGURE 3 | Environmental variables significantly associated with talaromycosis in younger ( $<50$ years) and older patients ( $\geq 50$ years).

with candidiasis (Table 1), which may explain why humidity remained a risk factor for talaromycosis hospitalizations of all subjects (Figure 1D). In the previous studies suggesting humidity as a predictor for talaromycosis, $27.1 \%$ (139/513) of the patients were with oral candidiasis, but the proportion of other types of candidiasis was not mentioned $(12,16)$. Hence, this hypothesis warrants further studies. Due to a small number of patients with comorbidities, diabetes $(n=21)$ and liver cirrhosis $(n=22)$, we did not evaluate the risk factors for these two populations.

By now, whether increased levels of air pollutants, e.g., $\mathrm{NO}_{2}$, $\mathrm{PM}_{10}$, and $\mathrm{SO}_{2}$, the well-known risk factors for various infectious diseases (30-33), also affect the incidence of talaromycosis remains unclear. In this study, we showed that an IQR increase in $\mathrm{SO}_{2}$ at lag 5-lag 7 was significantly associated with talaromycosis hospitalizations of patients with Pneumocystis pneumonia. Thus, for the first time, we revealed a link between air pollutants and talaromycosis pathogenesis. It should be noted that $\mathrm{SO}_{2}$ also represents a risk factor for Pneumocystis pneumonia hospitalizations of HIV-infected patients (17), and by now how Pneumocystis pneumonia would affect the incidence of talaromycosis is not fully understood. Given that these two co-infections are prevalent in HIV-infected patients, the pathogenesis of talaromycosis is likely more complex than previously thought and further investigations are required to explore the relationship between $\mathrm{SO}_{2}$, talaromycosis, and Pneumocystis pneumonia. It is also worth noting that $\mathrm{SO}_{2}$ is more likely a driven factor but not the cause for talaromycosis hospitalizations. Exposure to $\mathrm{SO}_{2}$ leads to irritation of airway, bronchoconstriction, and dyspnea (34), which may facilitate talaromycosis pathogenesis, and the mechanism requires further research.

In the age stratified study, we found temperature was the only variable positively associated with talaromycosis in both younger and older HIV-infected patients (Figure 3). Since the gender distribution, CD4 cell count, and frequency of co-infections and liver cirrhosis were similar in the two groups (Supplementary Table S7), these data suggested that the age variable does not significantly affect the kinds of risk factors for talaromycosis. Interestingly, we found pressure was negatively associated with talaromycosis hospitalizations of the older patients (Figure 3), and patients with pulmonary tuberculosis or chronic hepatitis co-infection (Figure 2), but the underlying mechanism was not clear and warrants further research.

In the present study, we employed a case-crossover design, which has been widely used for evaluating predictors influencing the short-term health results, particularly acute infectious diseases $(18,20,30,35-37)$, as an alternative to Poisson distribution model to explore the associations between environmental variables and talaromycosis hospitalizations, and successfully identified the specific meteorological variables and air pollutant associated with talaromycosis in HIV-infected patients (Figures 1D, 2), suggesting the feasibility of this method. Unlike previous studies using the counts of talaromycosis hospitalizations aggregated by week or by month (16), we here showed that acute exposure to the risk factors at a single day level (lag 0 -lag 7) was significantly associated with talaromycosis hospitalizations of HIV-infected patients, which would broaden our understanding of the epidemiology and pathogenesis of the opportunistic infection.

Our study is limited in that it was a single-center study and some patients with talaromycosis treated elsewhere were not included. However, since more than $80 \%$ of HIV-infected patients in Guangzhou were treated in Guangzhou Eighth People's Hospital according to Guangzhou CDC report (19), and a large sample size included in this study enabled overcoming this drawback and accurate assessment of the risk factors for talaromycosis.

In summary, our study confirmed an acute association between environmental variables and talaromycosis onset and also revealed the air pollutant as a specific risk factor for talaromycosis in a subpopulation with co-infection, which would 
undoubtedly provide clues for a greater understanding of the transmission and pathogenesis of the opportunistic infection prevalent in $\mathrm{HIV}$-infected patients.

\section{DATA AVAILABILITY STATEMENT}

The raw data supporting the conclusions of this article will be made available by the authors, without undue reservation.

\section{ETHICS STATEMENT}

The studies involving human participants were reviewed and approved by Ethics Committee of Guangzhou Eighth People's Hospital. Written informed consent for participation was not required for this study in accordance with the national legislation and the institutional requirements.

\section{AUTHOR CONTRIBUTIONS}

KD conceived the study and supervised all aspects of the study. YW and KD collected and analyzed the data and prepared the manuscript. All authors contributed to the article and approved the submitted version.

\section{FUNDING}

This study was supported by the Natural Science Foundation of Guangdong Province (2017A030310035).

\section{REFERENCES}

1. Supparatpinyo K, Khamwan C, Baosoung V, Nelson KE, Sirisanthana T. Disseminated Penicillium marneffei infection in southeast Asia. Lancet. (1994) 344:110-3. doi: 10.1016/S0140-6736(94)91287-4

2. Vanittanakom N, Cooper CR, Jr., Fisher MC, Sirisanthana T. Penicillium marneffei infection and recent advances in the epidemiology and molecular biology aspects. Clin Microbiol Rev. (2006) 19:95-110. doi: 10.1128/CMR.19.1.95-110.2006

3. Hu Y, Zhang J, Li X, Yang Y, Zhang Y, Ma J, et al. Penicillium marneffei infection: an emerging disease in mainland China. Mycopathologia. (2013) 175:57-67. doi: 10.1007/s11046-012-9577-0

4. Zheng J, Gui X, Cao Q, Yang R, Yan Y, Deng L, et al. A clinical study of acquired immunodeficiency syndrome associated Penicillium Marneffei infection from a non-endemic area in China. PLoS One. (2015) 10:e0130376. doi: 10.1371/journal.pone.0130376

5. Antinori S, Gianelli E, Bonaccorso C, Ridolfo AL, Croce F, Sollima $\mathrm{S}$, et al. Disseminated Penicillium marneffei infection in an HIVpositive Italian patient and a review of cases reported outside endemic regions. J Travel Med. (2006) 13:181-8. doi: 10.1111/j.1708-8305.2006. 00039.x

6. Patassi AA, Saka B, Landoh DE, Kotosso A, Mawu K, Halatoko WA, et al. First observation in a non-endemic country (Togo) of Penicillium marneffei infection in a human immunodeficiency virus-infected patient: a case report. BMC Res Notes. (2013) 6:506. doi: 10.1186/1756-0500-6-506

7. Huynh TX, Nguyen HC, Dinh Nguyen HM, Do MT, Odermatt-Biays S, Degremont A, et al. [Penicillium marneffei infection and AIDS. A review of 12 cases reported in the Tropical Diseases Centre, Ho Chi Minh City (Vietnam)]. Sante. (2003) 13:149-53. Available online at: https://www.jle.com/fr/revues/ san/e-docs/penicilliose_et_sida_a_propos_de_12_cas_diagnostiques_au_

\section{SUPPLEMENTARY MATERIAL}

The Supplementary Material for this article can be found online at: https://www.frontiersin.org/articles/10.3389/fmed. 2021.731188/full\#supplementary-material

Supplementary Figure $\mathbf{S 1}$ | Correlations between environmental variables. The Pearson correlation coefficient is shown in each cell.

Supplementary Figure $\mathbf{S 2}$ | Dynamics of relative count, humidity, and temperature during 2014-2019.

Supplementary Table S1 | Summary statistics for environmental variables in Guangzhou, 2014-2019.

Supplementary Table S2 | Associations between talaromycosis hospitalizations and an IQR increase in environmental variables.

Supplementary Table S3 | Associations between talaromycosis hospitalizations of HIV-infected patients with candidiasis and an IQR increase in environmental variables.

Supplementary Table S4 | Associations between talaromycosis hospitalizations of HIV-infected patients with Pneumocystis pneumonia and an IQR increase in environmental variables.

Supplementary Table S5 | Associations between talaromycosis hospitalizations of HIV-infected patients with pulmonary tuberculosis and an IQR increase in environmental variables.

Supplementary Table S6 | Associations between talaromycosis hospitalizations of HIV-infected patients with chronic hepatitis B or C and an IQR increase in environmental variables.

Supplementary Table S7 | Demographic and clinical features of patients stratified by age.

Supplementary Table S8 | Associations between talaromycosis hospitalizations of younger patients and an IQR increase in environmental variables.

Supplementary Table S9 | Associations between talaromycosis hospitalizations of older patients and an IQR increase in environmental variables.

centre_des_maladies_tropicales_ho_chi_minhville_vietnam_262840/ article.phtml

8. Cao C, Xi L, Chaturvedi V. Talaromycosis (Penicilliosis) Due to Talaromyces (Penicillium) marneffei: insights into the clinical trends of a major fungal disease 60 years after the discovery of the pathogen. Mycopathologia. (2019) 184:709-20. doi: 10.1007/s11046-019-00410-2

9. Jiang J, Meng S, Huang S, Ruan Y, Lu X, Li JZ, et al. Effects of Talaromyces marneffei infection on mortality of HIV/AIDS patients in southern China: a retrospective cohort study. Clin Microbiol Infect. (2019) 25:233-41. doi: 10.1016/j.cmi.2018.04.018

10. Limper AH, Adenis A, Le T, Harrison TS. Fungal infections in HIV/AIDS. Lancet Infect Dis. (2017) 17:e334-e43. doi: 10.1016/S1473-3099(17)30303-1

11. Larsson M, Nguyen LH, Wertheim HF, Dao TT, Taylor W, Horby P, et al. Clinical characteristics and outcome of Penicillium marneffei infection among HIV-infected patients in northern Vietnam. AIDS Res Ther. (2012) 9:24. doi: 10.1186/1742-6405-9-24

12. Le T, Wolbers M, Chi NH, Quang VM, Chinh NT, Lan NP, et al. Epidemiology, seasonality, and predictors of outcome of AIDS-associated Penicillium marneffei infection in Ho Chi Minh City, Viet Nam. Clin Infect Dis. (2011) 52:945-52. doi: 10.1093/cid/cir028

13. Huang X, He G, Lu S, Liang Y, Xi L. Role of Rhizomys pruinosus as a natural animal host of Penicillium marneffei in Guangdong, China. Microb Biotechnol. (2015) 8:659-64. doi: 10.1111/1751-7915.12275

14. Cao C, Liang L, Wang W, Luo H, Huang S, Liu D, et al. Common reservoirs for Penicillium marneffei infection in humans and rodents, China. Emerg Infect Dis. (2011) 17:209-14. doi: 10.3201/eid1702.100718

15. Chariyalertsak S, Sirisanthana T, Supparatpinyo K, Praparattanapan J, Nelson KE. Case-control study of risk factors for Penicillium marneffei infection in human immunodeficiency virus-infected patients in northern Thailand. Clin Infect Dis. (1997) 24:1080-6. doi: 10.1086/513649 
16. Bulterys PL, Le T, Quang VM, Nelson KE, Lloyd-Smith JO. Environmental predictors and incubation period of AIDS-associated Penicillium marneffei infection in Ho Chi Minh City, Vietnam. Clin Infect Dis. (2013) 56:1273-9. doi: $10.1093 / \mathrm{cid} / \mathrm{cit} 058$

17. Djawe K, Levin L, Swartzman A, Fong S, Roth B, Subramanian A, et al. Environmental risk factors for Pneumocystis pneumonia hospitalizations in HIV patients. Clin Infect Dis. (2013) 56:74-81. doi: 10.1093/cid/cis841

18. Wei Y, Wang Y, Di Q, Choirat C, Wang Y, Koutrakis P, et al. Short term exposure to fine particulate matter and hospital admission risks and costs in the Medicare population: time stratified, case crossover study. BMJ. (2019) 367:16258. doi: 10.1136/bmj.16258

19. Guangzhou Centers for Disease Control and Prevention. Available online at: http://www.gzcdc.org.cn/download/index.html (accessed May 8, 2021).

20. Maclure M. The case-crossover design: a method for studying transient effects on the risk of acute events. Am J Epidemiol. (1991) 133:144-53. doi: 10.1093/oxfordjournals.aje.a115853

21. Levy D, Lumley T, Sheppard L, Kaufman J, Checkoway H. Referent selection in case-crossover analyses of acute health effects of air pollution. Epidemiology. (2001) 12:186-92. doi: 10.1097/00001648-200103000-00010

22. Cheng CY, Cheng SY, Chen CC, Pan HY, Wu KH, Cheng FJ. Ambient air pollution is associated with pediatric pneumonia: a time-stratified case-crossover study in an urban area. Environ Health. (2019) 18:77. doi: 10.1186/s12940-019-0520-4

23. Pope CA, Muhlestein JB, Anderson JL, Cannon JB, Hales NM, Meredith $\mathrm{KG}$, et al. Short-term exposure to fine particulate matter air pollution is preferentially associated with the risk of ST-segment elevation acute coronary events. J Am Heart Assoc. (2015) 4:e002506. doi: 10.1161/JAHA.115.0 02506

24. Tsai SS, Yang CY. Fine particulate air pollution and hospital admissions for pneumonia in a subtropical city: Taipei, Taiwan. J Toxicol Environ Health A. (2014) 77:192-201. doi: 10.1080/15287394.2013.853337

25. Chang CC, Crane M, Zhou J, Mina M, Post JJ, Cameron BA, et al. HIV and co-infections. Immunol Rev. (2013) 254:114-42. doi: 10.1111/imr.12063

26. Bell LCK, Noursadeghi M. Pathogenesis of HIV-1 and Mycobacterium tuberculosis co-infection. Nat Rev Microbiol. (2018) 16:80-90. doi: $10.1038 /$ nrmicro.2017.128

27. Centers for Disease Control and Prevention. Available online at: https://www. cdc.gov/hiv/group/age/olderamericans/index.html (accessed June 2, 2021).

28. Wei W, He J, Ning C, Xu B, Lai J, Jiang J, et al. Maxent modeling for Predicting the Potential Distribution of Global Talaromycosis [Preprint]. Available online at: https://www.biorxiv.org/content/10.1101/2021.03.28.437430v1.full (accessed June 27, 2021).

29. Dou N, Li W, Zhao E, Wang C, Xiao Z, Zhou H. Risk factors for candida infection of the genital tract in the tropics. Afr Health Sci. (2014) 14:835-9. doi: 10.4314/ahs.v14i4.10
30. Alessandrini ER, Stafoggia M, Faustini A, Berti G, Canova C, De Togni A, et al. Association between short-term exposure to PM2.5 and PM10 and mortality in susceptible subgroups: a multisite case-crossover analysis of individual effect modifiers. Am J Epidemiol. (2016) 184:744-54. doi: 10.1093/aje/kww078

31. Grigg J. Air pollution and respiratory infection: an emerging and troubling association. Am J Respir Crit Care Med. (2018) 198:700-1. doi: 10.1164/rccm.201804-0614ED

32. Su W, Wu X, Geng X, Zhao X, Liu Q, Liu T. The short-term effects of air pollutants on influenza-like illness in Jinan, China. BMC Public Health. (2019) 19:1319. doi: 10.1186/s12889-019-7607-2

33. Huang K, Ding K, Yang XJ, Hu CY, Jiang W, Hua XG, et al. Association between short-term exposure to ambient air pollutants and the risk of tuberculosis outpatient visits: a time-series study in Hefei, China. Environ Res. (2020) 184:109343. doi: 10.1016/j.envres.2020.109343

34. Kampa M, Castanas E. Human health effects of air pollution. Environ Pollut. (2008) 151:362-7. doi: 10.1016/j.envpol.2007.06.012

35. Horne BD, Joy EA, Hofmann MG, Gesteland PH, Cannon JB, Lefler JS, et al. Short-term elevation of fine particulate matter air pollution and acute lower respiratory infection. Am J Respir Crit Care Med. (2018) 198:759-66. doi: 10.1164/rccm.201709-1883OC

36. Pirozzi CS, Jones BE, VanDerslice JA, Zhang Y, Paine R 3rd, Dean NC. Shortterm air pollution and incident pneumonia. A case-crossover study. Ann Am Thorac Soc. (2018) 15:449-59. doi: 10.1513/AnnalsATS.201706-495OC

37. Zhang Y, Ding Z, Xiang Q, Wang W, Huang L, Mao F. Short-term effects of ambient PM1 and PM2.5 air pollution on hospital admission for respiratory diseases: case-crossover evidence from Shenzhen, China. Int J Hyg Environ Health. (2020) 224:113418. doi: 10.1016/j.ijheh.2019.11.001

Conflict of Interest: The authors declare that the research was conducted in the absence of any commercial or financial relationships that could be construed as a potential conflict of interest.

Publisher's Note: All claims expressed in this article are solely those of the authors and do not necessarily represent those of their affiliated organizations, or those of the publisher, the editors and the reviewers. Any product that may be evaluated in this article, or claim that may be made by its manufacturer, is not guaranteed or endorsed by the publisher.

Copyright (c) 2021 Wang and Deng. This is an open-access article distributed under the terms of the Creative Commons Attribution License (CC BY). The use, distribution or reproduction in other forums is permitted, provided the original author(s) and the copyright owner(s) are credited and that the original publication in this journal is cited, in accordance with accepted academic practice. No use, distribution or reproduction is permitted which does not comply with these terms. 